\title{
Short 57 kb CDKN2A FISH probe effectively detects short homozygous deletion of the 9p21 locus in malignant pleural mesothelioma
}

\author{
YUZO OYAMA $^{1}$, MAKOTO HAMASAKI ${ }^{1}$, SHINJI MATSUMOTO ${ }^{1}$, \\ AYUKO SATO $^{2}$, TOHRU TSUJIMURA ${ }^{2}$ and KAZUKI NABESHIMA ${ }^{1}$ \\ ${ }^{1}$ Department of Pathology, Fukuoka University Hospital and School of Medicine, Jonan-ku, Fukuoka 814-0180; \\ ${ }^{2}$ Department of Pathology, Hyogo College of Medicine, Nishinomiya, Hyogo 663-8131, Japan
}

Received May 28, 2021; Accepted September 2, 2021

DOI: $10.3892 / \mathrm{ol} .2021 .13074$

\begin{abstract}
Homozygous deletion (homo-d) of the cyclin-dependent kinase inhibitor $2 \mathrm{~A}(C D K N 2 A)$ gene is frequently found in malignant pleural mesothelioma (MPM). Fluorescence in situ hybridization (FISH) is commonly used to detect chromosomal deletion, and sometimes reveals more frequent heterozygous deletion (hetero-d) compared with homo-d. In clinical practice, such $C D K N 2 A$ FISH results belong to the 'borderline' homo-d rate, which makes it difficult to definitively diagnose MPM. Microdeletion, [<200 kilobase (kb)], can induce a 'pseudo' hetero-d signal in FISH assays with long probes owing to redundant probe reactivity. Thus, the present study hypothesized that shorter FISH probes can effectively detect the small deletion status of the CDKN2A gene and increase homo-d rate in MPM, which has high hetero-d and low homo-d status. The present study aimed to evaluate the effectiveness of a shorter $C D K N 2 A$ FISH probe in diagnosing MPM. $C D K N 2 A$ FISH with either a $222 \mathrm{~kb}$ long probe (L-probe) or a $57 \mathrm{~kb}$ short probe (S-probe) was performed in four MPM cases with high hetero-d and low homo-d patterns. Furthermore, immunohistochemistry for methylthioadenosine phosphorylase (MTAP) and quantitative (q)PCR analyses were
\end{abstract}

Correspondence to: Dr Makoto Hamasaki, Department of Pathology, Fukuoka University Hospital and School of Medicine, 7-45-1 Nanakuma, Jonan-ku, Fukuoka 814-0180, Japan

E-mail: makotoha@fukuoka-u.ac.jp

Abbreviations: MPM, malignant pleural mesothelioma; FISH, fluorescence in situ hybridization; homo-d, homozygous deletion; IHC, immunohistochemistry; BAP1, BRCA1-associated protein 1; MTAP, methylthioadenosine phosphorylase; CDKN2A, cyclin-dependent kinase inhibitor $2 \mathrm{~A}$; $\mathrm{kb}$, kilobase; hetero-d, heterozygous deletion; qPCR, quantitative PCR; TBLB, transbronchial lung biopsy

Key words: malignant mesothelioma, CDKN2A, fluorescence in situ hybridization, microdeletion, MTAP, qPCR performed to confirm the microdeletion of the 9p21 locus. The results demonstrated that all four MPM cases retained MTAP protein expression. $C D K N 2 A$ FISH with L-probe revealed high hetero-d (cases 1-4; 73.3, 37.1, 59.2 and 64.8\%, respectively) and low homo-d (cases 1-4; 12.1, 12.4, 25.4 and 22.2\%, respectively). $C D K N 2 A$ FISH with S-probe revealed high homo-d (cases $1-4 ; 96.8,90.0,87.5$ and $82.6 \%$, respectively), with low hetero-d (cases 1-4; 0.0, 1.2, 1.2 and $4.3 \%$, respectively). qPCR analysis demonstrated no allele deletions of the MTAP gene and two-allele deletions of the $C D K N 2 A$ gene in $3 / 4$ cases. Taken together, these results suggest that the S-probe detects the short homo-d of the 9p21 locus more effectively than the L-probe in MPM. This can assist in solving diagnostic difficulties in cases involving high hetero-d with low homo-d.

\section{Introduction}

Malignant pleural mesothelioma (MPM) is an aggressive neoplasm that arises from mesothelial cells that line the serosal membrane of the pleura (1). MPM requires accurate and early diagnosis as early treatment improves patient prognosis (2). However, it is difficult to distinguish MPM from benign mesothelial proliferative disorders in routine practice due to overlaps in morphology (3). Thus, genomic-based ancillary assays, such as fluorescence in situ hybridization (FISH) to detect homozygous deletion (homo-d) of the 9p21 locus and immunohistochemistry (IHC) analysis to detect loss of BRCA1-associated protein 1 (BAP1) expression and methylthioadenosine phosphorylase [MTAP; the protein product of the MTAP gene located in the telomere side of cyclin-dependent kinase inhibitor 2A $(C D K N 2 A)$ in the 9p21 locus (3)] proteins, are effective tools for detecting characteristic genetic abnormalities that are essential in the diagnosis of MPM $(3,4)$.

The $C D K N 2 A$ gene, located at the 9p21 locus (5), is a tumor suppressor gene which encodes the p16 protein (5). Homo-d of $C D K N 2 A$ is one of the most frequent genetic abnormalities in malignant mesothelioma and is found in 50-65.8\% of MPM cases (6,7). $C D K N 2 A$ FISH is a common assay used to detect this deletion, and a 222 kilobase (kb) CDKN2A probe (long probe, L-probe) is a commercially available and the most frequently used FISH probe, which covers an area of $222 \mathrm{~kb}$, 
including the $C D K N 2 A, C D K N 2 B$ and MTAP genes. Another commercially available probe is the $57 \mathrm{~kb} C D K N 2 A$ probe (short probe, $\mathrm{S}$-probe) that covers an area of $57 \mathrm{~kb}$, confined to the $C D K N 2 A$ and $C D K N 2 B$ genes (6,7). FISH reveals heterozygous deletion (hetero-d) signals in addition to homo-d and normal signals (8). Recently, $C D K N 2 A$ FISH with L-probe revealed high proportions of hetero-d, while $S$-probe yielded high proportions of homo-d, with low hetero-d (8).

Microdeletion, defined as a deletion $<200 \mathrm{~kb}$, can induce false negative results of signal deletion in FISH assays, owing to redundant probe reactivity (9-13). Some of the high hetero-d signals may result from microdeletions in MPM (8). It was hypothesized that an S-probe can effectively detect microdeletion status of the $C D K N 2 A$ gene, and thus increase homo-d rate in MPM, which has a high hetero-d and low homo-d status with L-probe (8).

The present study aimed to evaluate the effectiveness of $\mathrm{S}$-probe in diagnosing MPM. In routine practice, it is important to confirm homo-d of the CDKN2A gene for an accurate diagnosis of MPM, to start treatment and predict prognosis. Borderline cases are infrequently found in which FISH with L-probe reveals high hetero-d and low homo-d; however, it is worth performing FISH with S-probe if homo-d of CDKN2A is detected (2). Thus, the aforementioned hypothesis was investigated in four cases of MPM, in which CDKN2A FISH with L-probe yielded high hetero-d and low homo-d. The results of FISH with S-probe were assessed in association with the deletion range evaluated via quantitative (q)PCR analysis.

\section{Materials and methods}

Case selection. CDKN2A FISH results were reviewed in 106 MPM cases that were diagnosed at the Department of Pathology of Fukuoka University Hospital (Fukuoka, Japan) between January 2017 and December 2019, including consultation cases. A total of five cases of MPM exhibited high hetero-d and low homo-d pattern in $C D K N 2 A$ FISH. A total of four cases were used in the present study, owing to the presence of redundant tissue, for further investigation. All four samples were obtained from male patients, the mean age at diagnosis was 68.5 years (age range, 63-80 years). Pathological diagnoses were performed according to the 2021 World Health Organization classification of thoracic tumors (1). The mesothelial origin was confirmed by positive reactivity for Wilms tumor 1, calretinin or podoplanin (D2-40) and negative reactivity for epithelial cell adhesion molecule (BerEP4), thyroid transcription factor-1, claudin-4 or carcinoembryonic antigen. The present study was approved by the Institutional Review Board of Fukuoka University (approval no. 11-7-11). The requirement for informed consent was waived as the anonymous use of redundant tissues is part of the standard treatment agreement with patients, when no objection is expressed.

IHC staining. For immunostaining, 4- $\mu$ m-thick sections of formalin-fixed paraffin embedded (FFPE) tissues were used. Sections were deparaffinized and rehydrated with xylene, alcohol and tap water.IHC analysis was performedon an automatedDAKO Omnis platform using the DAKO EnVision FLEX, High $\mathrm{pH}$ (Omnis https://www.agilent.com/ja-jp/product/dako-omnis-solution-for-ihc-ish/dako-omnis/dako-omnis-75902, code GV800), according to the manufacturer's instructions. Heat-epitope retrieval was performed using a high $\mathrm{pH}$ for $30 \mathrm{~min}$ at $97^{\circ} \mathrm{C}$. Protein blocking was performed using Protein block Serum-Free (Agilent Technologies, Inc.) for $20 \mathrm{~min}$ at $25^{\circ} \mathrm{C}$. The slides were subsequently incubated using one of the following antibodies: Mouse monoclonal anti-calretinin antibody (clone Calret1, cat. no. M7245, Dako; Agilent Technologies, Inc., ready to use; room temperature for $40 \mathrm{~min}$ ), mouse monoclonal anti-BAP1 antibody (clone C-4, cat. no. sc-28383, Santa Cruz Biotechnology, Inc.; 1:100 dilution; room temperature for $1 \mathrm{~h}$ ) or mouse monoclonal anti-MTAP antibody (clone 2G4, cat. no. H00004507-M01J, Abnova; 1:100 dilution; room temperature for $45 \mathrm{~min}$ ). Endogenous peroxidase was inactivated using peroxidase blocking reagent (Dako; Agilent Technologies, Inc.) for $3 \mathrm{~min}$ at room temperature. Subsequently, slides were incubated with a peroxidase-labeled polymer (EnVision Flex/HRP, cat. no. DM842; Agilent Technologies, Inc.), at room temperature for $20 \mathrm{~min}$ ). The slides were visualized with 3,3'-diaminobenzidine at room temperature for $5 \mathrm{~min}$ and counterstained with hematoxylin at room temperature for $5 \mathrm{~min}$. Analysis was performed using an Olympus BX 53 light microscope (magnification, $\mathrm{x} 400$ ). Each specimen was evaluated by two authors ( $\mathrm{YO}$ and $\mathrm{KN}$ ).

BAP1 and MTAP staining results were scored as loss or retained. BAP1 loss in tumor cells was defined as complete loss of nuclear staining. MTAP loss in tumor cells was defined as complete loss of cytoplasmic staining in the area where internal positive control cells (lymphocytes, histiocytes, fibroblasts, pneumocytes or endothelial cells) exhibited cytoplasmic and nuclear MTAP staining (14).

FISH assay. CDKN2A FISH was performed using 4- $\mu$ m-thick FFPE tissue sections. Sections were deparaffinized, rehydrated in 100, 90 and $70 \%$ ethanol, washed with $2 x$ saline sodium citrate (SSC) (UltraPure ${ }^{\mathrm{TM}}$ 20X SSC, Invitrogen; Thermo Fisher Scientific, Inc.), incubated in pretreatment solution (PathVysion HER2 DNA probe kit; $30 \mathrm{~min}$ at $80^{\circ} \mathrm{C}$ ), and digested with pepsin solution (Sigma-Aldrich; Merck $\mathrm{KGaA}$, for $3 \mathrm{~h}$ at $37^{\circ} \mathrm{C}$ ). Refixation was performed using $10 \%$ formalin (10 $\mathrm{min}$ at room temperature); sections were subsequently washed with 2x SSC, dehydrated in ethanol, dried and exposed to either of two DNA probe: $222 \mathrm{~kb}$ Vysis LSI CDKN2A/CEP9 probe alone (Abbott Japan LLC) or 57 kb SureFISH 9p21.3 CDKN2A (Agilent Technologies, Inc.) probe and $367 \mathrm{~kb}$ SureFISH Chr9 CEP FISH probe (Agilent Technologies, Inc,). Denaturation $\left(10 \mathrm{~min}\right.$ at $\left.80^{\circ} \mathrm{C}\right)$ and hybridization $\left(24 \mathrm{~h}\right.$ at $\left.37^{\circ} \mathrm{C}\right)$ were performed in ThermoBrite (Abbott Japan LLC.). After $24 \mathrm{~h}$, the specimens were treated with $2 x$ SSC containing $0.3 \%$ NP 40 (Nonidet P-40, nacalai tesque) $\left(2 \mathrm{~min}\right.$ at $\left.72^{\circ} \mathrm{C}\right), 2 \mathrm{x}$ SSC containing $0.1 \%$ NP 40 (5 min at room temperature) and $2 \mathrm{x} \mathrm{SSC} \mathrm{(5} \mathrm{min} \mathrm{at} \mathrm{room} \mathrm{temperature).}$ Nuclei were counterstained with DAPI using antifade reagent for $24 \mathrm{~h}$ at $4^{\circ} \mathrm{C}$ (VECTASHIELD Mounting Medium, Vector Laboratories, Inc.). Analysis was performed using a fluorescence microscope (magnification, $\mathrm{x} 1,000)$ (Axio imager Z1; Carl Zeiss AG) and Isis FISH imaging system (Metasystems, https://metasystems-international.com/en/products/isis). FISH signal was classified under three signal patterns: Normal (two red and two green signals), hetero-d (one red and two green signals) and homo-d (no red and two green signals), and $>100$ tumor cells were assessed for each case. 
Table I. Primer sets.

\begin{tabular}{|c|c|c|c|c|}
\hline Set & Primer & Sequence $\left(5^{\prime}-3^{\prime}\right)$ & Target region (gene) & Product size (bp) \\
\hline \multirow[t]{2}{*}{ A } & $\mathrm{F}$ & GGGCACTTTGTGACTCTTCTTAACC & \multirow[t]{2}{*}{ MTAP } & \multirow[t]{2}{*}{105} \\
\hline & $\mathrm{R}$ & GGAGACTTTGGGGTATGGTCCTC & & \\
\hline \multirow[t]{2}{*}{ B } & $\mathrm{F}$ & TACAGGTAGGGAAGCAGGCAATC & Non-coding region between & \multirow[t]{2}{*}{90} \\
\hline & $\mathrm{R}$ & TATGGGTATGCTCTGGTCTTCTGC & $M T A P$ and $C D K N 2 A$ & \\
\hline \multirow[t]{2}{*}{$\mathrm{C}$} & $\mathrm{F}$ & GCGATTGCTGCCAAGATACCC & Non-coding region between & \multirow[t]{2}{*}{89} \\
\hline & $\mathrm{R}$ & TCCACAGGCCTTTGTCTCCAG & $M T A P$ and $C D K N 2 A$ & \\
\hline \multirow[t]{2}{*}{$\mathrm{D}$} & $\mathrm{F}$ & TTCCGGACACACTGGGTCAC & Non-coding region between & \multirow[t]{2}{*}{99} \\
\hline & $\mathrm{R}$ & GTTTACAGGCAAGTGAGGTTGTCC & $M T A P$ and $C D K N 2 A$ & \\
\hline \multirow[t]{2}{*}{$\mathrm{E}$} & $\mathrm{F}$ & GCCTGTTTTCTTTCTGCССTCTGC & \multirow[t]{2}{*}{$C D K N 2 A$} & \multirow[t]{2}{*}{89} \\
\hline & $\mathrm{R}$ & GACTGATGATCTAAGTTTCCCGAGGTTTCTC & & \\
\hline \multirow[t]{2}{*}{$\mathrm{F}$} & $\mathrm{F}$ & CCACATCTTCACGCCTTCGC & \multirow[t]{2}{*}{$C D K N 2 A$} & \multirow[t]{2}{*}{83} \\
\hline & $\mathrm{R}$ & AGGGTTGCAAGAAGAAAACGAGTG & & \\
\hline \multirow[t]{2}{*}{ RPS6 } & $\mathrm{F}$ & CTACTGAGTAAGGGGCATTCCTGT & \multirow[t]{2}{*}{ The reference gene } & \multirow[t]{2}{*}{102} \\
\hline & $\mathrm{R}$ & GAGAACGCTCAGATTTGCATCCAC & & \\
\hline
\end{tabular}

F, forward; R, reverse; bp, base pair.

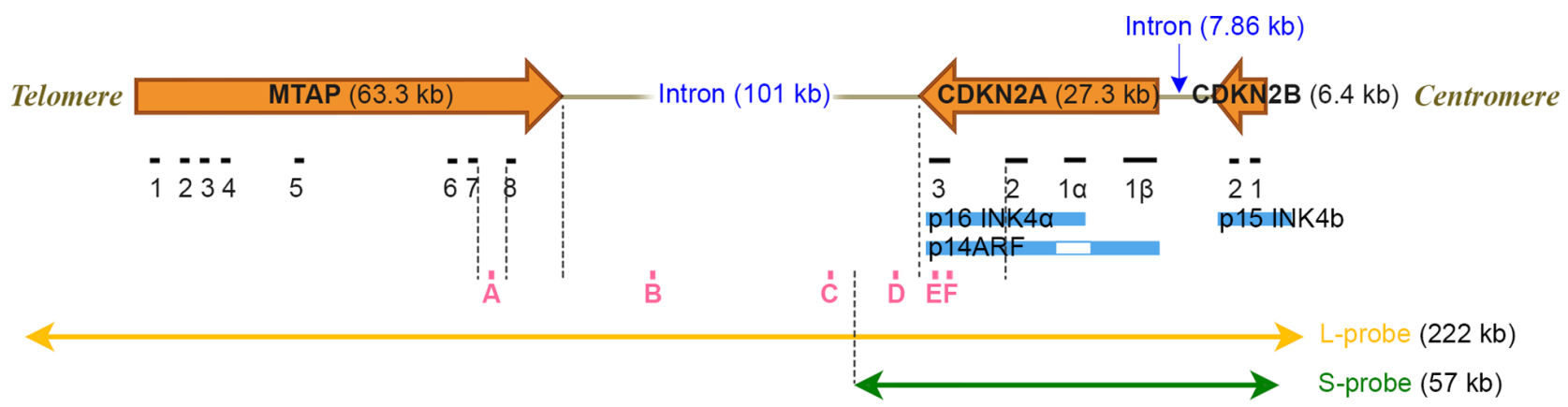

Figure 1. Chromosome map of the 9p21 region and CDKN2A FISH probe targeting areas. The L-probe covers areas up to $222 \mathrm{~kb}$, which includes the $C D K N 2 A$, $C D K N 2 B$ and $M T A P$ genes. The S-probe only covers areas up to $57 \mathrm{~kb}$, which includes the $C D K N 2 A$ and $C D K N 2 B$ genes. A, B, C, D, E and F are the locations of each PCR product used in quantitative PCR analysis. CDKN, cyclin-dependent kinase inhibitor; MTAP, methylthioadenosine phosphorylase; L-probe, long probe; S-probe, short probe.

DNA extraction. A total of 6/7 sections of 10- $\mu \mathrm{m}$ FFPE-cut specimens were used for each case. Before extracting DNA, macrodissection was performed to purify the tumor samples, with the exception of case 4 where the sample was too small. Genomic DNA was extracted using a RecoverAllTM Total Nucleic Acid Isolation kit (Ambion; Thermo Fisher Scientific, Inc.; cat. no. AM1975), according to the manufacturer's instructions, with one modification: Protease digestion was performed for 20-24 h. To confirm the quality of extracted DNA samples, the positive control ( $\beta$-globin) primer (forward, 5'-ACACAACTGTGTTCACTAGC-3' and reverse, 5'-CAACTTCATCCACGTTCACC-3') region was amplified via PCR using KOD FX Neo (Toyobo, Ltd., cat. no. KFX-201). The PCR reaction protocol was as follows: $94^{\circ} \mathrm{C}$ for $15 \mathrm{~min}$, followed by 35 cycles of denaturation at $98^{\circ} \mathrm{C}$ for $10 \mathrm{sec}$, annealing at $55^{\circ} \mathrm{C}$ for $30 \mathrm{sec}$, and extension at $68^{\circ} \mathrm{C}$ for $30 \mathrm{sec}$. The PCR products were detected by electrophoresis of $3 \%$ agarose gel with GelRed ${ }^{\mathrm{TM}}$ Nucleic Acid Gel Stain $(10,000 x)$ in DMSO (Biotium, Inc.), visualized by ultraviolet light. $q P C R$. The extracted DNA from tumor tissue samples was used for $\mathrm{qPCR}$. The chromosome map of the $9 \mathrm{p} 21$ region and the targeting regions of the two commercially available $C D K N 2 \mathrm{~A}$ FISH probes are presented in Fig. 1. The seven primer sets for analyzing the deleted region and the reference gene region were designed by custom oligo primers (Sigma-Aldrich; Merck KGaA) (Table I). Primer A was designed for the MTAP gene, primers B-D were designed for the non-coding region between the $C D K N 2 A$ and MTAP genes and primers $\mathrm{E}$ and $\mathrm{F}$ were designed for the $C D K N 2 A$ gene. The copy number of the regions was quantified via qPCR analysis of genomic DNA using KOD SYBR ${ }^{\circledR}$ qPCR Mix (Toyobo Life Science). The PCR reaction protocol was as follows: $98^{\circ} \mathrm{C}$ for $15 \mathrm{~min}$, followed by 40 cycles of denaturation at $98^{\circ} \mathrm{C}$ for $10 \mathrm{sec}$, annealing at $60^{\circ} \mathrm{C}$ (primer A, primer F, RPS6) or $62^{\circ} \mathrm{C}$ (primers B, C, D and E) for $10 \mathrm{sec}$, and extension at $68^{\circ} \mathrm{C}$ for $30 \mathrm{sec}$. Melting curve analysis was also performed for non-specific products. The copy number was determined by the relative standard curve method using the RPS6 gene (9p22) (15) as the reference gene according to the previous reports $(9,15)$, and the calculated 
Table II. Clinicopathological characteristics of the four MPM cases assessed in the present study.

\begin{tabular}{|c|c|c|c|c|}
\hline Characteristic & Case 1 & Case 2 & Case 3 & Case 4 \\
\hline Age, years & 62 & 78 & 68 & 66 \\
\hline Sex & Male & Male & Male & Male \\
\hline Histology & Epithelioid & Epithelioid & Epithelioid & Epithelioid \\
\hline MTAP IHC & Retained & Retained & Retained & Retained \\
\hline BAP1 IHC & Loss & Retained & Loss & Loss \\
\hline Source of tissue & $\mathrm{EPP}$ & Pleural biopsy & Pleural biopsy & TBLB \\
\hline Treatment & $\begin{array}{l}\text { EPP + radiation; } \\
\text { CDDP + PEM; } \\
\text { GEM + VNR }\end{array}$ & CBDCA + PEM & Chemotherapy & None \\
\hline Time from diagnosis to mortality & 7 years and 6 months & 2 years and 2 months & 5 months & 3 months \\
\hline
\end{tabular}

MPM, malignant pleural mesothelioma; MTAP, methylthioadenosine phosphorylase; BAP1, BRCA1-associated protein 1; IHC, immunohistochemistry; EPP, extrapleural pneumonectomy; TBLB, transbronchial lung biopsy; CDDP, cisplatin; PEM, pemetrexed sodium hydrate; GEM, gemcitabine hydrochloride; VNR, vinorelbine detartrate; CBDCA, carboplatin.

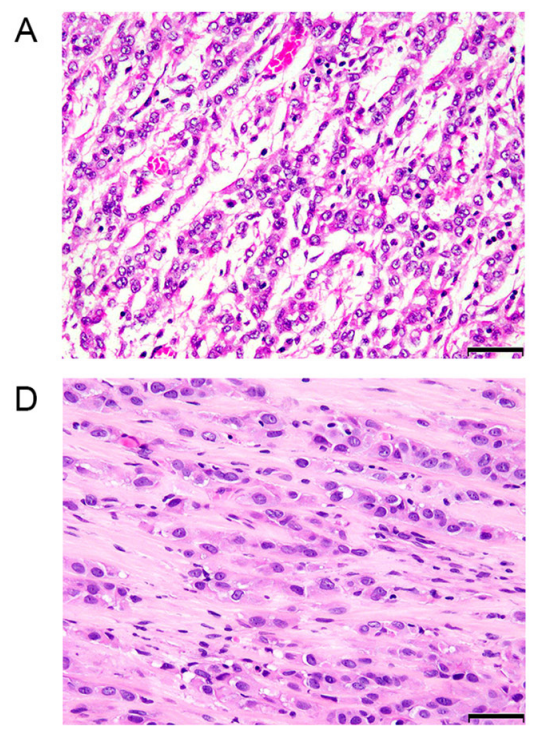

G

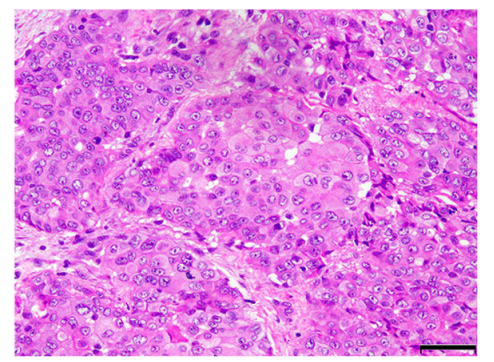

$J$

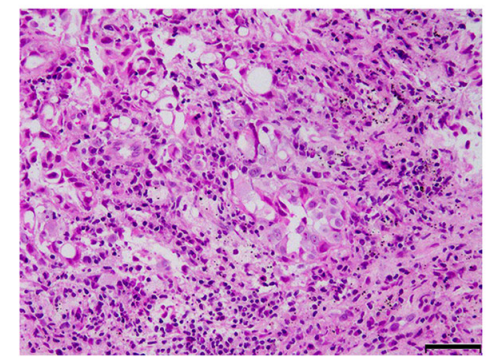

B

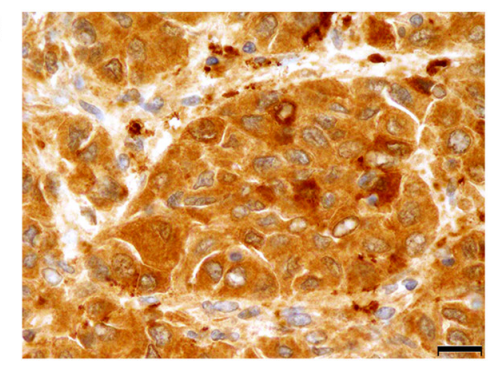

E

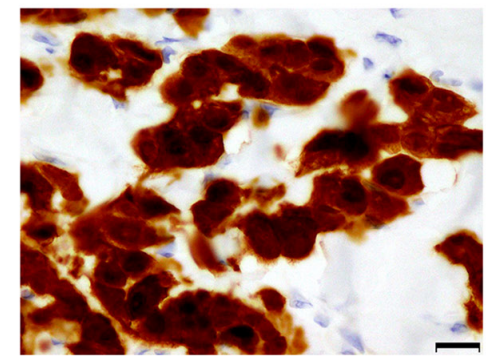

$\mathrm{H}$

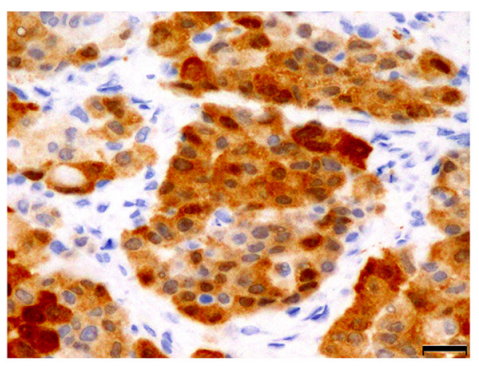

K

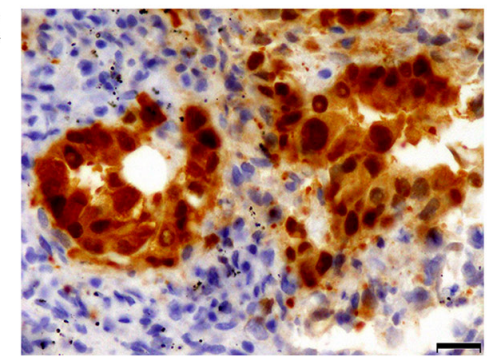

C

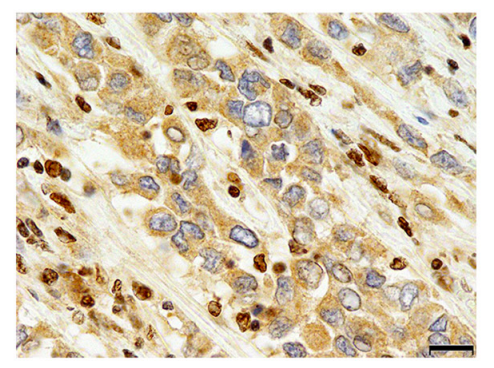

F
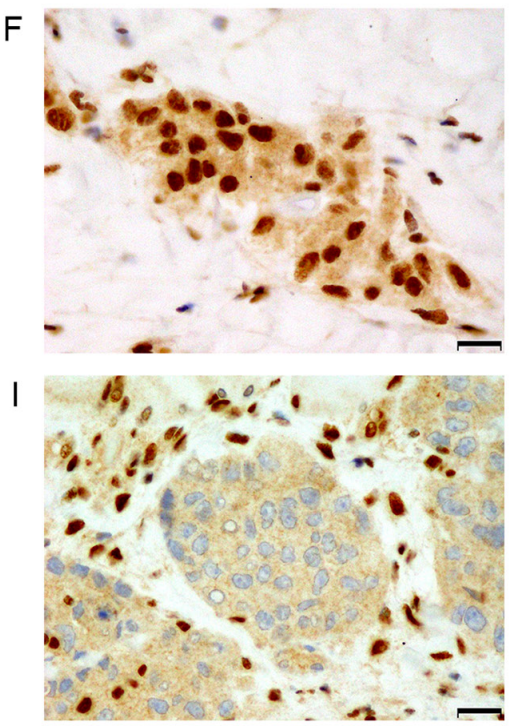

L

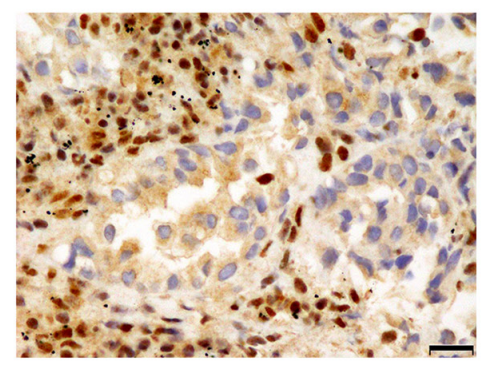

Figure 2. Histology of malignant pleural mesothelioma (A-C, case 1; D-F, case 2; G-I, case 3 and J-L, case 4). (A, D, G and J) The tumor exhibited proliferation of atypical epithelioid tumor cells with eosinophilic cytoplasm, arranged in cords or small nests. (B, E, H and K) Tumor cells were positive for calretinin. (C, I and L) Cases 1, 3 and 4 exhibited BAP1 loss, (F) whereas case 2 retained BAP1 expression. Olympus BX 53 light microscope. (A, D, G and J) Magnifications: x200 scale bar, $50 \mu \mathrm{m}$ ). (B, C, E, F, H, I, K and L) Magnification: x400 scale bar, $20 \mu \mathrm{m}$. BAP1, BRCA1-associated protein 1. 


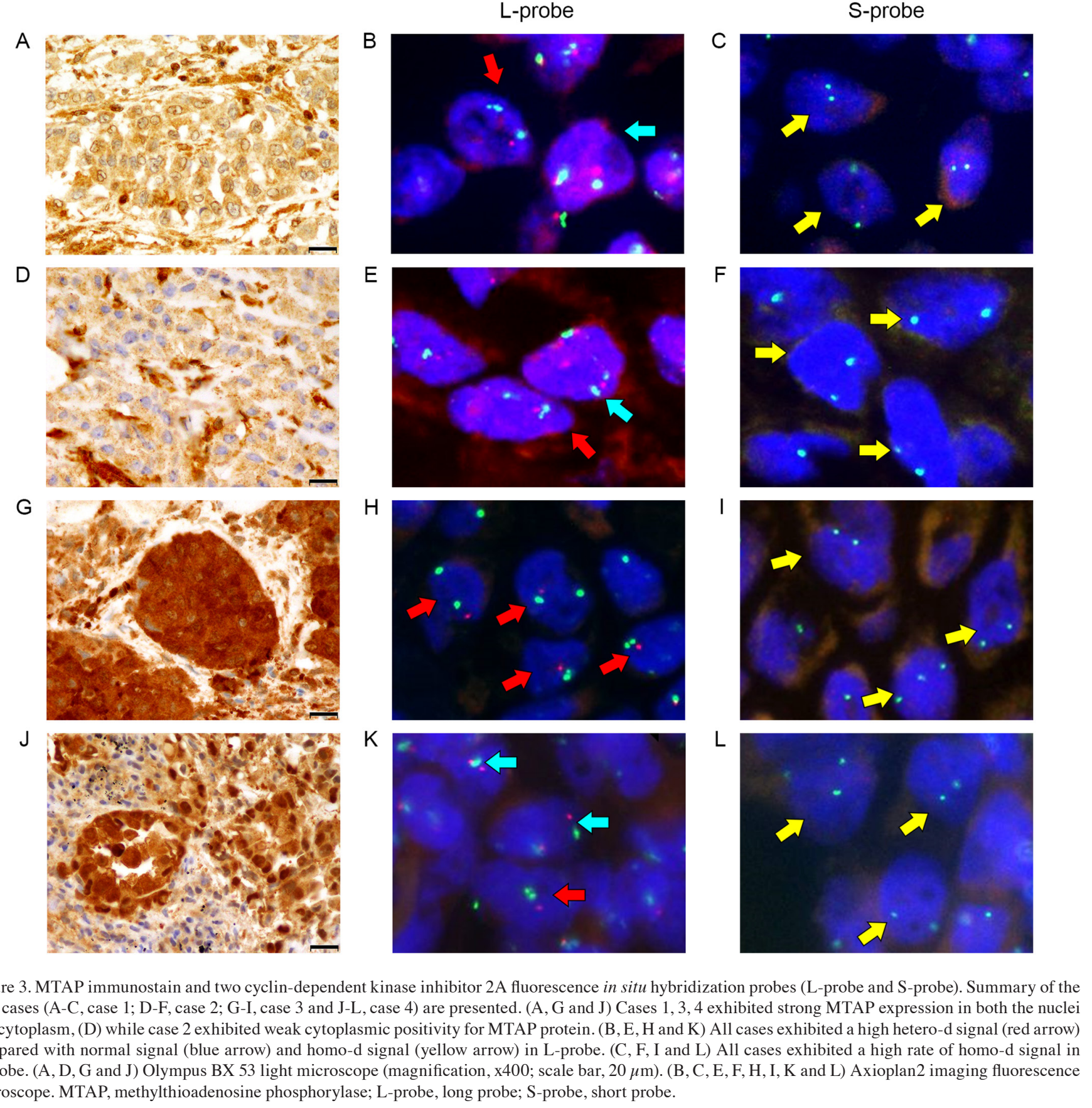

Figure 3. MTAP immunostain and two cyclin-dependent kinase inhibitor 2A fluorescence in situ hybridization probes (L-probe and S-probe). Summary of the four cases (A-C, case 1; D-F, case 2; G-I, case 3 and J-L, case 4) are presented. (A, G and J) Cases 1, 3, 4 exhibited strong MTAP expression in both the nuclei and cytoplasm, (D) while case 2 exhibited weak cytoplasmic positivity for MTAP protein. (B, E, H and K) All cases exhibited a high hetero-d signal (red arrow) compared with normal signal (blue arrow) and homo-d signal (yellow arrow) in L-probe. (C, F, I and L) All cases exhibited a high rate of homo-d signal in S-probe. (A, D, G and J) Olympus BX 53 light microscope (magnification, x400; scale bar, $20 \mu \mathrm{m}$ ). (B, C, E, F, H, I, K and L) Axioplan2 imaging fluorescence microscope. MTAP, methylthioadenosine phosphorylase; L-probe, long probe; S-probe, short probe.

numbers were evaluated as follows: $\geq 0.8$ as no deletion, $>0.5$ and $<0.8$ as a one-allele deletion, and $\leq 0.5$ as a two-allele deletion.

\section{Results}

Clinicopathological characteristics. The clinicopathological characteristics are summarized in Table II. All four samples were obtained from male patients, the mean age at diagnosis was 68.5 years (age range, 63-80 years). A total of two samples were obtained following pleural biopsy, one following extrapleural pneumonectomy and one following transbronchial lung biopsy (TBLB) from a metastatic lesion. All cases were epithelioid MPM in terms of histological subtype (Fig. 2A, D, G and J). Mesothelial phenotype was confirmed by positive immunos- taining for calretinin (Fig. 2B, E, H and K). IHC analysis of BAP1 protein (Fig. 2C, F, I and L) was as follows: Three cases with BAP1 loss and one with BAP1 retained. The mean period from diagnosis to mortality was 2.6 years (3-90 months).

MTAP IHC and CDKN2A FISH results. A total of 3/4 cases exhibited strong nuclear and cytoplasmic or cytoplasmic only expression for MTAP protein (Fig. 3A, G and J), while case 2 exhibited relatively weak cytoplasmic reactivity for MTAP (Fig. 3D). With the L-probe, CDKN2A FISH revealed high hetero-d signals in all cases (cases 1-4; 73.3, 37.1, 59.2 and $64.8 \%$, respectively) (Fig. 3B, E, H and K) compared with homo-d signals (cases 1-4; 12.1, 12.4, 25.4 and 22.2\%, respectively). In the hetero-d signals with L-probe, one red signal was slightly smaller than two CEP9 green signals. 


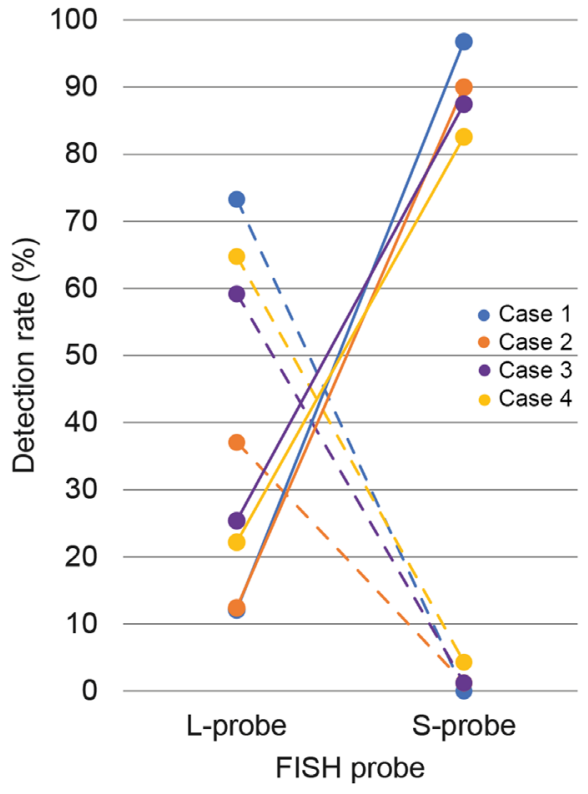

Figure 4. Comparative line graph of homo- and hetero-d rates between the Land S-probes. The vertical line represents the detection rate of homo-d (solid line) and hetero-d (dashed line) signals, while the horizontal line represents the corresponding results of L- and S-probes. For the L-probe, the hetero-d rate was high compared with the homo-d signal for each case. However, the homo-d rate increased by the S-probe. L-probe, long probe; S-probe, short probe; homo-d, homozygous deletion; hetero-d; heterozygous deletion; FISH, fluorescence in situ hybridization.

However, the $C D K N 2 A$ FISH with S-probe exhibited high homo-d in all cases (cases 1-4; 96.8, 90.0, 87.5 and 82.6\%, respectively) (Fig. 3C, F, I and L) compared with low hetero-d (cases $1-4 ; 0.0 \%, 1.2,1.2$ and $4.3 \%$, respectively). In the control tissues, $C D K N 2 A$ FISH with S-probe exhibited normal signals (Fig. S1). The comparative line graph of homo-d (solid line) and hetero-d (dotted line) demonstrated the increased proportions of homo-d and decreased proportions of hetero-d in FISH with S-probe compared with L-probe (Fig. 4).

$q P C R$. qPCR analysis revealed no allele deletions in the MTAP gene in all cases, whereas two-allele deletions were detected in the $C D K N 2 A$ gene in $3 / 4$ cases (Fig. 5). Case 4 exhibited one-allele deletion in the $C D K N 2 A$ gene. Deletion status of the non-coding region between the $C D K N 2 A$ and MTAP genes varied, two-allele deletions for case 2 and one-allele deletion for cases 1,3 and 4, respectively.

\section{Discussion}

To the best of our knowledge, the preset study was the first to demonstrate that $C D K N 2 A$ FISH with S-probe is more effective in elucidating short homo-d in the 9p21 locus compared with L-probe in MPM. CDKN2A FISH with conventional L-probe revealed high hetero-d (range 37.1-73.3\%) and low homo-d (range 12.1-25.4\%) in all four cases with microdeletions, whereas that with S-probe yielded high proportions of homo-d (82.6-96.8\%). Furthermore, qPCR analysis in these four cases demonstrated that $3 / 4$ cases exhibited homo-d in the coding region of the $C D K N 2 A$ gene, while case 4 exhibited hetero-d, according to the calculated value. In case 4 , two-allele deletions were not confirmed via qPCR analysis
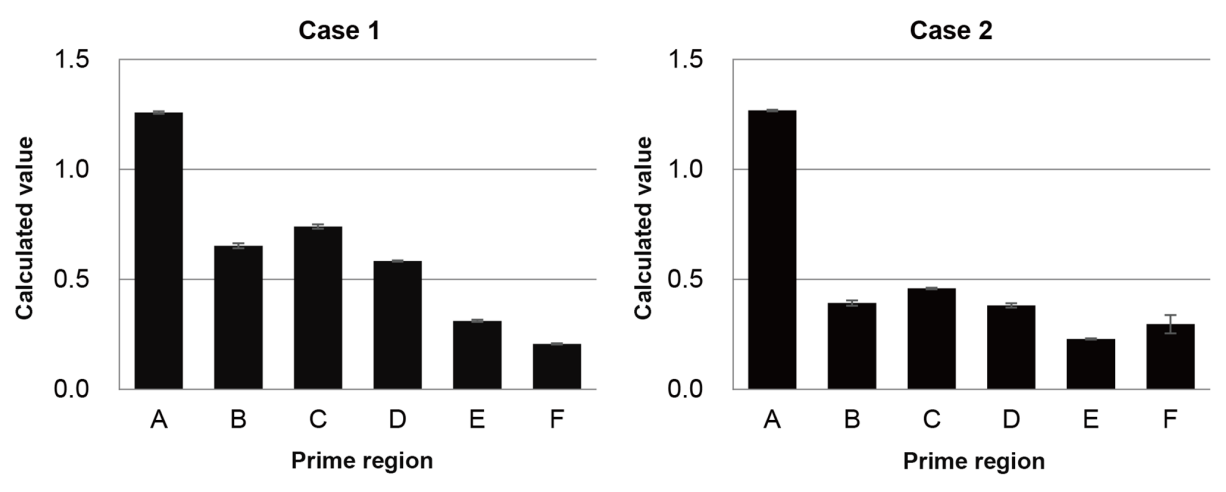

Case 3
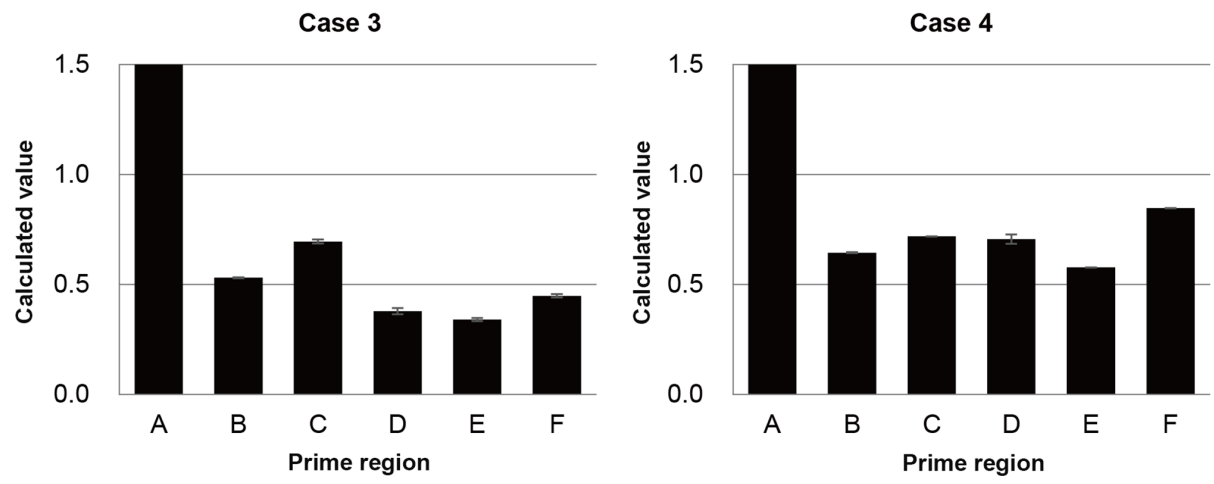

Figure 5. qPCR analysis in the 9p21 locus. qPCR analysis was performed to detect the deletion status of the 9p21 regions. The MTAP gene (primer A) had no allele deletion, while two-allele deletions were observed for the $C D K N 2 A$ gene (primer E and F) in all cases except for case 4 . Deletion status of the non-coding region between the CDKN2A and MTAP genes (primer B-D) was two- or one-allele deletion for each case. qPCR, quantitative PCR; MTAP, methylthioadenosine phosphorylase; $C D K N 2 A$, cyclin-dependent kinase inhibitor $2 \mathrm{~A}$. 
despite the clear evidence of homo-d exhibited by FISH as the TBLB section was too small to allow macrodissection prior to qPCR. Thus, it was speculated that contamination of normal cells would affect the qPCR results. Based on these results, the size of deletions was $\sim 27.3-142.5 \mathrm{~kb}$ at most. The results of the present study suggest it is worth performing $C D K N 2 A$ FISH with S-probe when $C D K N 2 A$ FISH with conventional L-probe results in high hetero-d and low homo-d.

The deletion size of the 9p21 locus found in MPM is usually 2-3 megabase in in vitro studies using cell lines; this range is large enough to accommodate the MTAP gene, as well as the $C D K N 2 A$ and $C D K N 2 B$ genes (16). However, in tumor specimens, the deleted region varies in size and range $(9,10)$. Microdeletion in 9p21.3 is found in 32\% of cases of acute lymphoblastic leukemia in adolescents and $10 \%$ of Ewing sarcoma $(9,10)$. The smallest deletion is only $25 \mathrm{~kb}$ on the $C D K N 2 A$ gene (10). Cases with $C D K N 2 A$ microdeletion fail to exhibit high proportions of homo-d by FISH with L-probe, as the L-probe, which is designed to cover three genes in the 9p21 locus (CDKN2A, CDKN2B and MTAP) (10), is large enough to hybridize the non-deleted area next to the microdeleted CDKN2A gene, resulting in 'pseudo' hetero-d signals $(9,13)$. In the present study, the false red signals were smaller compared with normal CEP9 green signals. Given the design of the L-probe and respective gene sizes, it is understandable that microdeletion cases exhibited high hetero-d and low homo-d with L-probe in $C D K N 2 A$ FISH.

In the present study, MTAP expression was retained in all four cases. MTAP IHC is a useful surrogate assay for $C D K N 2 A$ FISH as homo-d of MTAP always occurs in association with homo-d of $C D K N 2 A(6,14)$. However, it would be favorable to perform $C D K N 2 A$ FISH in cases with retained MTAP expression determined by IHC as retained MTAP protein expression does not necessarily rule out a microdeletion status of the CDKN2A gene $(8,14)$.

Detection of $C D K N 2 A$ deletion is important in routine practice as it effectively discriminates MPM from reactive mesothelial proliferations, and is associated with poor prognosis in MPM (3-6). There are several approaches used to circumvent the false negative results in detecting chromosomal microdeletion, including use of smaller probes (as used in the present study), enhancement of FISH signals (13), multiple ligation-dependent probe amplification (MLPA) assay (11) and array comparative genomic hybridization (CGH) (10). Practical use of S-probe can be somewhat challenging as the target red signal is small owing to the short probe length (9); thus, lower hybridization efficiency can result in false positive results for $C D K N 2 A$ deletion (9). Therefore, careful establishment of an accurate FISH assay, which always reveals two red signals and two green signals in normal cells, is critical.

The present study is not without limitations. First, the sample size was too small. Prospective studies including S-probe performance in several MPM cases, that have high hetero-d and low homo-d status with L-probe, are warranted to verify the effectiveness of S-probe. Secondly, the S-probe can cause false negative results of signal deletion if the deletion range is too small (9-13). On such occasions, enhancement of FISH signals, MLPA assay and array CGH can be alternative approaches to detect $C D K N 2 A$ gene deletion if MPM is highly suspected.
In the single institutional cohort assessed, $4.7 \%$ of MPM cases exhibited microdeletion of the $C D K N 2 A$ gene, with high hetero-d and low homo-d detected by FISH with the conventional L-probe. The use of $C D K N 2 A$ FISH with a short $57 \mathrm{~kb}$ probe can be an effective approach to detect a small homo-d in MPM. Furthermore, S-probe can increase the homo-d rate in MPM cases with high hetero-d with L-probe, and thus assist in solving diagnostic difficulties in cases involving high hetero-d with low homo-d.

\section{Acknowledgements}

The authors would like to thank Ms Hiroyo Fukagawa (Department of Pathology, Fukuoka University Hospital and School of Medicine, Fukuoka, Japan) for her assistance with qPCR analysis.

\section{Funding}

The present study was supported by grants from the Research Center for Advanced Molecular Medicine, Fukuoka University (grant no. 171032), the Ministry of the Environment (grant nos. 190557 and 200598) and Grants-in Aid for Scientific Research (grant no. 24590495) from the Japanese Society for the Promotion of Science.

\section{Availability of data and materials}

The datasets used and/or analyzed during the current study are available from the corresponding author upon reasonable request.

\section{Authors' contributions}

YO performed the experiments, drafted and revised the manuscript and analyzed the data. MH conceptualized and supervised the present study, and drafted and revised the manuscript. SM curated the data, acquired the data, analyzed and interpreted the data, and drafted and revised the manuscript. AS and TT acquired the resources, curated the data, acquired the data, and analyzed and interpreted the data, and drafted and revised the manuscript. KN conceptualized and supervised the present study, was project administrator, acquired funding, and drafted and revised the manuscript. $\mathrm{YO}, \mathrm{MH}$, and $\mathrm{KN}$ confirmed the authenticity of all the raw data. All authors have read and approved the final manuscript.

\section{Ethics approval and consent to participate}

The present study was approved by the Institutional Review Board of Fukuoka University (Fukuoka, Japan; approval no. 11-7-11). The requirement for informed consent was waived as the anonymous use of redundant tissues is part of the standard treatment agreement with patients, when no objection is expressed.

\section{Patient consent for publication}

Not applicable. 


\section{Competing interests}

The authors declare that they have no competing interests.

\section{References}

1. Sauter JL, Kadota K, Bueno R, Ladanyi M, Dacic S, Nowak AK, Gill RR, Schmitt F and Husain AN: Diffuse pleural mesothelioma. In: WHO Classification of Tumours: thoracic Tumours. IARC Press, Lyon, pp204-219, 2021.

2. Rosen LE, Karrison T, Ananthanarayanan V, Gallan AJ, Adusumilli PS, Alchami FS, Attanoos R, Brcic L, Butnor KJ, Galateau-Sallé F, et al: Nuclear grade and necrosis predict prognosis in malignant epithelioid pleural mesothelioma: A multi-institutional study. Mod Pathol 31: 598-606, 2018.

3. Chapel DB, Schulte JJ, Husain AN and Krausz T: Application of immunohistochemistry in diagnosis and management of malignant mesothelioma. Transl Lung Cancer Res 9 (Suppl 1): S3-S27, 2020.

4. Kinoshita Y, Hamasaki M, Matsumoto S, Yoshimura M, Sato A, Tsujimura T, Kamei T, Kawahara K and Nabeshima K: Genomic-based ancillary assays offer improved diagnostic yield of effusion cytology with potential challenges in malignant pleural mesothelioma. Pathol Int 70: 671-679, 2020.

5. Illei PB, Rusch VW, Zakowski MF and Ladanyi M: Homozygous deletion of CDKN2A and codeletion of the methylthioadenosine phosphorylase gene in the majority of pleural mesotheliomas. Clin Cancer Res 9: 2108-2113, 2003.

6. Sheffield BS, Hwang HC, Lee AF, Thompson K, Rodriguez S, Tse CH, Gown AM and Churg A: BAP1 immunohistochemistry and p16 FISH to separate benign from malignant mesothelial proliferations. Am J Surg Pathol 39: 977-982, 2015.

7. Yoshimura M, Kinoshita Y, Hamasaki M, Matsumoto S, Hida T, Oda Y, Iwasaki A and Nabeshima K: Highly expressed EZH2 in combination with BAP1 and MTAP loss, as detected by immunohistochemistry, is useful for differentiating malignant pleural mesothelioma from reactive mesothelial hyperplasia. Lung Cancer 130: 187-193, 2019.

8. Hamasaki M, Matsumoto S, Abe S, Hamatake D, Kamei T, Hiroshima K, Kawahara K, Sato A, Tsujimura T, Nakatani Y, et al: Low homozygous/high heterozygous deletion status by p16 FISH correlates with a better prognostic group than high homozygous deletion status in malignant pleural mesothelioma. Lung Cancer 99: 155-161, 2016.
9. Savola S, Nardi F, Scotlandi K, Picci P and Knuutila S: Microdeletions in 9p21.3 induce false negative results in CDKN2A FISH analysis of Ewing sarcoma. Cytogenet Genome Res 119: 21-26, 2007.

10. Usvasalo A, Savola S, Räty R, Vettenranta K, Harila-Saari A, Koistinen P, Savolainen ER, Elonen E, Saarinen-Pihkala UM and Knuutila S: CDKN2A deletions in acute lymphoblastic leukemia of adolescents and young adults: An array CGH study. Leuk Res 32: 1228-1235, 2008.

11. Martinez Ciarpaglini C, Gonzalez J, Sanchez B, Agusti J, Navarro L, Nieto $G$ and Monteagudo C: The amount of melanin influences p16 loss in spitzoid melanocytic lesions: Correlation with CDKN2A status by FISH and MLPA. Appl Immunohistochem Mol Morphol 27: 423-429, 2019.

12. Tsutsumi Y, Chinen Y, Sakamoto N, Nagoshi H, Nishida K, Kobayashi S, Yokokawa Y, Taki T, Sasaki N, Yamamoto-Sugitani M, et al: Deletion or methylation of CDKN2A/2B and PVT1 rearrangement occur frequently in highly aggressive B-cell lymphomas harboring 8q24 abnormality. Leuk Lymphoma 54: 2760-2764, 2013.

13. Mizuno Y, Chinen Y, Tsukamoto T, Takimoto-Shimomura T, Matsumura-Kimoto Y, Fujibayashi Y, Kuwahara-Ota S, Fujino T, Nishiyama D, Shimura Y, et al: A novel method of amplified fluorescent in situ hybridization for detection of chromosomal microdeletions in B cell lymphoma. Int J Hematol 109: 593-602, 2019.

14. Hida T, Hamasaki M, Matsumoto S, Sato A, Tsujimura T, Kawahara K, Iwasaki A, Okamoto T, Oda Y, Honda H, et al: Immunohistochemical detection of MTAP and BAP1 protein loss for mesothelioma diagnosis: Comparison with 9p21 FISH and BAP1 immunohistochemistry. Lung Cancer 104: 98-105, 2017.

15. Sato A, Torii I, Tao LH, Song M, Kondo N, Yoshikawa Y, Hashimoto-Tamaoki T, Hasegawa S, Nakano T and Tsujimura T: Establishment of a cell line from a Japanese patient useful for generating an in vivo model of malignant pleural mesothelioma. Cancer Sci 102: 648-655, 2011.

16. Cheng JQ, Jhanwar SC, Klein WM, Bell DW, Lee WC, Altomare DA, Nobori T, Olopade OI, Buckler AJ and Testa JR: p16 alterations and deletion mapping of 9p21-p22 in malignant mesothelioma. Cancer Res 54: 5547-5551, 1994. 